\title{
The PBS-12SF Cell Line: Development of an Alternative Cell Line for Influenza Vaccine Production
}

\author{
Kelly RB Sporer ${ }^{*}$, Jenna L. Carter and Paul M Coussens \\ Molecular Pathogenesis Laboratory, Department of Animal Science, Michigan State University, East Lansing, MI 48824, USA \\ *Corresponding author: Kelly R.B. Sporer 474 South Shaw Lane 3270E Anthony Hall, East Lansing, MI 48824, USA, Tel: 517-355-8443; Fax: 517-353-1699; E-mail: \\ buckhamk@msu.edu
}

Received date: November 01, 2016; Accepted date: November 04, 2016; Published date: November 09, 2016

Copyright: (c) 2016 Sporer KRB, et al. This is an open-access article distributed under the terms of the Creative Commons Attribution License, which permits unrestricted use, distribution, and reproduction in any medium, provided the original author and source are credited.

\begin{abstract}
Influenza viruses cause global seasonal epidemics with the potential to cause widespread illness and mortality. Vaccination is the single best approach to prevent serious illness caused by influenza infection; however, the current egg-based vaccination technology possesses many drawbacks despite being a robust method for over 60 years. Cell culture-based vaccine production systems possess many advantages over the current method. Over the last ten years, our research group has developed and improved upon an immortalized chick embryo cell line that has shown promise as a possibility for influenza vaccine production. The purpose of this short communication is to briefly review the development of cell lines to produce influenza vaccines, focusing on our group's work with the PBS-1 and PBS-12SF lines.
\end{abstract}

\section{Cell Lines in Influenza Vaccine Production}

Seasonal influenza outbreaks are responsible for millions of infections every year that lead to hospitalizations and deaths, especially in vulnerable subsets of the human population, such as infants, the elderly and pregnant women. Serious illness is best prevented by vaccination as treatments may be limited in availability, like neuraminidase inhibitors, or not effective, like adamantanes [1]. Of great concern is the ever-present possibility of a human transmissible avian influenza or a highly virulent $\mathrm{H} 1 \mathrm{~N} 1$ pandemic.

Since the 1940s, influenza vaccines have been produced with the utilization of embryonated chicken eggs. The advantages and disadvantages of the current egg-based production system have been extensively reviewed [1-3]. Briefly, the egg-based system has been adapted over time to grow influenza virus to high titers and has been scaled up to an automated and standardized system that has proven to be safe and effective in protecting most individuals from seasonal influenza. However, its numerous drawbacks include: time and laborintensiveness, dependency on egg availability in huge quantities, contamination in downstream processing, mutations/lack of viral stability when passaged in eggs, and the inability to administer these vaccines to egg-allergic individuals. Additionally, not all influenza strains grow well in eggs $[1,4]$. All of these factors may result in the failure to protect the human population during a pandemic.

Numerous advantages exist to adopting a cell culture-based influenza vaccine production system as opposed to the utilization of embryonated chicken eggs. Continuous cell lines have already been successfully used to produce rabies and polio vaccines. Cell lines may be better characterized and easily scaled up to produce large volumes of virus in a standardized and controlled manufacturing process, may grow certain viruses, especially reassortant influenza viruses, to higher titers than eggs, and may be stored long term $[2,4]$. Additionally, there appears to be greater stability of virus propagated in cultured cells, resulting in vaccines that are more similar to circulating infectious influenza virus than those grown in eggs [2]. Requirements of cell lines for use in influenza virus production should include: free of serum or other animal-derived components, fast cell growth (doubling times of 20-30 h), high cell viability, easy scale-up, and fast virus production to high titers at a low multiplicity of infection (MOI) [4,5]. A number of cell-based vaccines are currently available. These include: Flucelvax Norvartis Vaccines and Diagnostics Unlimited), which is produced using Madin-Darby Canine Kidney (MDCK) cells, Prefucel and Celvapan (Baxter), produced using African green monkey (Vero) cells, and the FluBlok vaccine (Protein Sciences Corporation), which has been created using a baculovirus /insect cell method $[3,6]$. Other cell lines of interest or in testing include: PER.C6 cells, a human embryonic retinal cell line, the AGE.CR and EB66 lines, derived from duck embryonic retinal cells and duck embryonic stem cells, respectively, and the immortalized chick embryo cell line, PBS-1 [2].

\section{PBS-1 cells: susceptible to influenza}

Our group's work began with the parental cell line, CHCC-OU2, that was immortalized by $\mathrm{N}$-methyl-N'-nitro-N-nitrosoguanidine (MNNG) treatment [7]. The PBS-1 cells were selected from this parental cell line to exhibit rapid growth and susceptibility to a wide range of viruses, were non-tumorigenic, and were not prone to contaminating pathogens. Previously, we demonstrated that PBS-1 cells were, in fact, susceptible to numerous strains of both human and avian influenza viruses (Table 1) and could grow several of them to high titers $\left(10^{7} \mathrm{PFU} / \mathrm{ml}\right)$. We primarily utilized two methods for measuring virus replication. These were the well-established hemagglutination (HA) assay, involving the addition of serially diluted infected cell culture supernatants to chicken red blood cells and monitoring for agglutination. We also measured the $50 \%$ tissue culture infective dose $\left(\mathrm{TCID}_{50}\right)$ [8] by the addition of serially diluted infected cell culture supernatants to cultured cells and then visually observing cytopathic effect. We also presented several advantages of PBS-1 cells over other common cell lines, such as MDCK and Vero cells. PBS-1 cells did not require exogenous proteases such as tosyl phenylalanyl 
Page 2 of 3

chloromethyl ketone (TPCK)-treated trypsin, for adsorption of influenza viruses on the cells to reach the same or higher titers [9].

\begin{tabular}{|c|c|c|c|}
\hline Influenza strain & $\begin{array}{l}\text { Susceptible } \\
\text { species }\end{array}$ & Subtype & Source \\
\hline A/NewCaledonia/20/1999a,b & Human & H1N1 & $\mathrm{CDC}$ \\
\hline A/HongKong/2652/2006 ${ }^{a}$ & Human & H1N1 & $\mathrm{CDC}$ \\
\hline A/Hiroshima/52/2005 & Human & H3N2 & $\mathrm{CDC}$ \\
\hline A/Wisconsin/67/2005 a,b & Human & $\mathrm{H} 3 \mathrm{~N} 2$ & $\mathrm{CDC}$ \\
\hline $\mathrm{A} / \mathrm{PR} / 8 / 34^{\mathrm{a}}$ & Human & H1N1 & ATCC \\
\hline A/Aichi/2/68 & Human & $\mathrm{H} 3 \mathrm{~N} 2$ & ATCC \\
\hline Alice ${ }^{a}$ & Human & $\mathrm{H} 3 \mathrm{~N} 2$ & ATCC \\
\hline B/Florida/07/2004a & Human & & $\mathrm{CDC}$ \\
\hline B/Malaysia/2506/2004a & Human & & $\mathrm{CDC}$ \\
\hline B/HongKong $/ 5 / 72^{a}$ & Human & & ATCC \\
\hline A/WhooperSwan/Mongolia/244/05 & Avian & H5N1 & $\begin{array}{l}\text { USDA } \\
\text { Endemic } \\
\text { Poultry } \\
\text { Viral } \\
\text { Disease } \\
\text { Research } \\
\text { Unit }\end{array}$ \\
\hline Anhui/01/2005-PR8 IBCDC RG-5 & Reassortant & & $\mathrm{CDC}$ \\
\hline VNH5N1-PR8/CDC-RGb & Reassortant & & $\mathrm{CDC}$ \\
\hline
\end{tabular}

Table 1: Influenza strains utilized to infect PBS- ${ }^{\mathrm{a}}$ and/or PBS- $12 \mathrm{SF}^{\mathrm{b}}$ cells.

\section{Generation of PBS-12SF line}

Our group next wished to adapt our influenza-susceptible PBS-1 cells to serum-free conditions. In this way, we could eliminate any animal products in the growth of our cells and avoid the serum lot-tolot variation and risk of contamination by viruses, mycoplasmas, and prions. We successfully achieved this through seven total passages of PBS-1 cells using decreasing percentages of serum-supplemented Dulbecco's Modified Eagle Medium (DMEM) and increasing percentages of Opti-Pro serum-free media supplemented with glutamine (Gluta- MAX-1; ThermoFisher Scientific), and antibiotics/ antifungals. Additionally, PBS-12SF cells were shown by flow cytometry to bind both sambucus nigra agglutinin (SNA) and maackia amurensis agglutinin (MAA) when incubated with the DIG-labeled lectins. This demonstrated that the cells expressed surface glycoproteins containing both the sialyl-galactose moieties Sia2- 6Gal, which preferentially binds human influenza viruses, and Sia2-3Gal, which preferentially binds avian influenza viruses. Subsequently, PBS-12SF cells were then shown to be susceptible to several strains of human and reassortant $\mathrm{H} 5 \mathrm{~N} 1$ influenza viruses (shown in Table 1) and to replicate these viruses to high titers comparable to or higher than MDCK, Vero, and primary chick embryo kidney (CEK) cells. Additionally, PBS-12SF cells, as previously shown in PBS-1 cells, did not require the addition of TPCK-treated trypsin in order to successfully replicate human and reassortant influenza virus [10].

\section{Knockdown of IFNAR1 in PBS-12SF Cells}

It has been established that Vero cells do not mount an interferonmediated response to viral infection [11]. We discovered that PBS-12SF cells mounted a Type-1 interferon (IFN)- mediated antiviral response when infected with human $(\mathrm{H} 1 \mathrm{~N} 1)$ or reassortant $(\mathrm{H} 5 \mathrm{~N} 1)$ influenza virus as demonstrated by an upregulation in both IFN- $\alpha$ and- $\beta$, as well as other important antiviral genes. Thus, we next set out to increase viral replication in PBS-12SF cells by attenuating their natural antiviral response through knockdown of the IFN- $\alpha / \beta$ receptor, IFNAR1. We successfully accomplished this by the design, production, and transfection of short hairpin RNA (shRNA)-expressing plasmids. shRNA-transfected PBS-12SF cells were proven to dramatically downregulate expression of IFNAR1 mRNA and protein. We then investigated the 6 relative expression of the important antiviral genes: 2'-5' oligoadenylate synthetase (OAS), interferon response factor 3 (IRF3), and interferon-induced helicase $\mathrm{C}$ domain containing 1(IFIH1) in our knockdown cells in response to chicken IFN- $\alpha$ (chIFNa) treatment. Expression of all three genes was dramatically upregulated by chIFNa treatment, and these results were significantly attenuated when the IFNAR1 was knocked down compared to the parental PBS-12SF cell line. An HA assay, comparison of hemagglutinin (HA) gene abundance, and immunostaining of influenza A H1N1 nucleoprotein (NP) in A/NewCaledonia/20/1999-infected control PBS-12SF and shRNA-transfected cells confirmed that cell infection rate was greater in IFNAR1-knockdown cells [12]. This reduced interferon response in our PBS-12SF line may give them another advantage as an alternative cell line for influenza vaccine production.

\section{Current and Future Work}

The standard method for evaluating stability of influenza viruses in cell culture as well as eggs involves serially passing virus in culture and obtaining the sequence of the hemagglutinin (HA) and neuraminidase (NA) genes at various passage levels. In order to demonstrate the genetic stability of influenza virus grown in PBS-12SF cells, we infected cells at an MOI of 0.1 then assessed for virus infectivity and collected culture supernatants at $48 \mathrm{~h}$ post-infection. Viral infectivity was measured by seeding PBS-12SF cells in a 96-well culture plate and applying serially diluted infected supernatant for $2 \mathrm{~h}$. Infection media was then replaced with complete media+4\% Tryp-LE Express for $48 \mathrm{~h}$. Cells were fixed with $4 \%$ paraformaldehyde, then stained using a mouse anti- influenza A nucleoprotein (NP) antibody and the VECTOR Red Alkaline Phosphatase (AP) Substrate Kit and Vectastain ABC-AP kit (Vector Laboratories). Microscopic visual evaluation of red (infected) cells was recorded and followed by TCID50 calculation [8]. At each passage, viral RNA was extracted from cell culture supernatants.

Reverse transcription was performed, and the entire HA and NA genes of the A/NewCaledonia/20/1999 strain were amplified using three sets of custom primers per gene; PCR amplicons were purified then sent for sequencing at Michigan State University's Research Technology Support Facility (RTSF). Preliminary sequencing results suggest genetic stability of both genes through three passages in PBS-12SF cells; however, these results are still being validated.

Additionally, our group has set out to create a biallelic knockout of the IFNAR1 gene in our PBS-12SF cells. We have custom designed CompoZr ${ }^{\mathrm{TM}}$ Knockout Zinc Finger Nucleases (ZFN; Sigma-Aldrich) and successfully transfected them into PBS-12SF cells. Validation of 
Citation: Sporer KRB, Carter JL, Coussens PM (2016) The PBS-12SF Cell Line: Development of an Alternative Cell Line for Influenza Vaccine

Page 3 of 3

the knockout is ongoing; and increased influenza viral production in IFNAR1-knockout cells is yet to be determined.

\section{Conclusion}

A cell-culture based influenza vaccine development system would have numerous advantages over the current widespread egg-based practice. We have developed and continued to improve upon an avian cell line, PBS-12SF that shows promise as a cell line candidate for influenza vaccine production.

\section{Acknowledgments}

We would like to acknowledge funding through the Michigan State University Targeted Support for Technology Development program. We also gratefully acknowledge the Center for Disease Control and HepaLife Technologies, Inc., as well as the contributions of Monica Carvajal-Yepes, Kristen Pabst, Christopher Colvin, Patty S.D. Weber, and Sue Sipkovsky.

\section{Financial Support}

Michigan State University Targeted Support for Technology Development program.

\section{References}

1. Nafziger AN, Pratt DS (2014) Seasonal influenza vaccination and technologies. J Clin Pharmacol 54: 719-731.

2. Hegde NR (2015) Cell culture-based influenza vaccines: A necessary and indispensable investment for the future. Hum Vaccin Immunother 11: 1223-1234.
3. Milian E, Kamen AA (2015) Current and emerging cell culture manufacturing technologies for influenza vaccines. Biomed Res Int pp: 504831.

4. Genzel Y, Reichl U (2009) Continuous cell lines as a production system for influenza vaccines. Expert Rev Vaccines 8: 1681-1692.

5. Vlecken DH, Pelgrim RP, Ruminski S, Bakker WA, van der Pol LA (2013) Comparison of initial feasibility of host cell lines for viral vaccine production. J Virol Methods 193: 28-41.

6. Song JM (2016) Advances in novel influenza vaccines: a patent review. J Microbiol 54: 403-412.

7. Ogura H, Fujiwara T (1987) Establishment and characterization of a virus-free chick cell line. Acta Med Okayama 41: 141-143.

8. Reed LJ, Muench H (1938) A simple method of estimating fifty per cent endpoints. Am J Hygiene 27: 493-497.

9. Smith KA, Colvin CJ, Weber PS, Spatz SJ, Coussens PM (2008) High titer growth of human and avian influenza viruses in an immortalized chick embryo cell line without the need for exogenous proteases. Vaccine 26: 3778-3782.

10. Coussens PM, Smith KA, Weber PS, Colvin CJ (2011) Immortalized chick embryo cell line adapted to serum-free growth conditions and capable of replicating human and reassortant H5N1 influenza strains for vaccine production. Vaccine 29: 8661-8668.

11. Desmyter J, Melnick JL, Rawls WE (1968) Defectiveness of interferon production and of rubella virus interference in a line of African green monkey kidney cells (Vero). J Virol 2: 955-961.

12. Carvajal-Yepes M, Sporer KR, Carter JL, Colvin CJ, Coussens PM (2015) Enhanced production of human influenza virus in PBS-12SF cells with a reduced interferon response. Hum Vaccin Immunother 11: 2296-2304. 